\title{
SAÚDE MENTAL DOS PROFISSIONAIS DE ENFERMAGEM QUE ATUAM NO COMBATE À COVID-r9
}

\author{
MENTAL HEALTH OF NURSING PROFESSIONALS WHO WORK TO COMBAT \\ COVID- 19
}

Letícya de Souza Olegário Duarte ${ }^{1}$

Annabelle de Fátima Modesto Vargas ${ }^{2}$

RESUMO: A Síndrome Respiratória Aguda causada pelo vírus SARS- CoV- 2, agente etiológico da doença Covid- 19, tem sido responsável por um cenário de grande tensão nos últimos 2 anos. Diante disso, surgiram os efeitos psicológicos da pandemia do novo coronavírus, principalmente entre os profissionais de Enfermagem, quais são o foco desta pesquisa. Este estudo objetiva analisar os principais fatores relacionados ao esgotamento psicológico e os meios de suporte à saúde mental desses profissionais. Para isso, foi realizada uma revisão integrativa baseada em 13 artigos encontrados na base de dados SciELO e nas plataformas Google Scholar, Ministério da Saúde e Conselho Federal de Enfermagem. Foram identificados que os principais efeitos colaterais decorrentes da pandemia são ansiedade, estresse, sentimento de impotência e medo, o que possui grande influência na saúde mental, tornando extremamente necessário meios de assistência psicológica que se adequem ao ambiente pandêmico. Nesse sentido, é possível perceber que a Covid- I9 compromete bem mais do que a saúde física de quem se depara com ela, também está relacionada com questões emocionais. Logo, é fundamental promover acesso a redes de apoio psicológico, além de melhorias no ambiente de trabalho.

Palavras-chave: Enfermagem. Saúde Mental. Covid- 19. Profissionais de Enfermagem. Pandemia.

ABSTRACT: The Acute Respiratory Syndrome caused by the SARS-CoV-2 virus, the etiologic agent of the Covid-I9 disease, has been responsible for a scenario of great tension in the last 2 years. Therefore, the psychological effects of the new coronavirus pandemic emerged, especially among nursing professionals, which are the focus of this research. This study aims to analyze the main factors related to

\footnotetext{
Graduanda de Enfermagem pela Uniredentor Centro Universitário. E-mail: leticyaduarte98@gmail.com

${ }^{2}$ Dr$^{\text {a }}$ Sociologia Política- UENF, Docente de Enfermagem da Uniredentor Centro Universitário. Email: annabelle.vargas@uniredentor.edu.br
} 
psychological exhaustion and the means to support these professionals' mental health. For this, an integrative review was carried out based on 13 articles found in the SciELO database and Google Scholar platforms, Ministry of Health and Federal Council of Nursing. It was identified that the main side effects resulting from the pandemic are anxiety, stress, feeling of impotence and fear, which has a great influence on mental health, making psychological assistance that adapt to the pandemic environment extremely necessary. In this sense, it is possible to see that Covid-19 compromises much more than the physical health of those who face it, it is also related to emotional issues. Therefore, it is essential to promote access to psychological support networks, in addition to improvements in the work environment.

Keywords: Nursing. Mental Health. Covid-19. Nursing Workers. Pandemic.

\section{INTRODUÇÃO}

A Covid- 19, doença causada pelo coronavírus intitulado de SARS-CoV-2, foi descoberta pela primeira vez na província de Wuhan, na China, em dezembro de 2019. No dia 30 de janeiro de 2020, a Organização Mundial de Saúde (OMS) anunciou que a epidemia de Covid- I9 estabelecia uma Emergência de Saúde Pública de Importância Internacional (ESPII), e, em II de março uma pandemia. Em I8 de março de 2020, os casos comprovados já haviam ultrapassado $214 \mathrm{mil} \mathrm{em} \mathrm{todo} \mathrm{mundo}$ (OLIVEIRA et al., 2020; FREITAS et al., 2020). Sendo, atualmente, de acordo com Werneck \& Carvalho (2020), um dos maiores desafios sanitários deste século.

A Síndrome Respiratória Aguda (SARS- Cov-2), é uma infecção respiratória potencialmente grave causada pelo novo coronavírus. As manifestações clínicas, geralmente, se assemelham desde um resfriado comum leve à uma pneumonia viral grave, podendo a intensidade dos sintomas variar, levando a uma síndrome de desconforto respiratório agudo, porventura, fatal (BEECHING $\mathrm{N}$ et al., 202I), além de possuir uma alta taxa de transmissibilidade.

O vírus é transmitido através de gotículas respiratórias, sendo eliminado nas secreções quando o indivíduo contaminado tosse, espirra ou fala, podendo contaminar outro indivíduo caso entre em contato direto com as membranas mucosas. A infecção também ocorre caso haja contato com superfícies contaminadas como celulares, mesas, talheres, entre outros e, posteriormente com os olhos, nariz e boca (BVS, 2020). "Os sintomas característicos incluem febre, tosse, dispneia e perda do paladar/olfato, no entanto, alguns pacientes podem ter sintomas leves do trato respiratório 
superior ou ser assintomáticos. As complicações da doença grave incluem, mas não são limitadas a falência de múltiplos órgãos, choque séptico e tromboembolismo venoso. Os sintomas podem ser persistentes e continuar por mais que 12 semanas em alguns pacientes" (BEECHING $\mathrm{N}$ et al., 2021, p. 3).

O combate à Covid- 19, dentro das unidades de saúde, demanda uma variedade de profissionais que inclui trabalhadores da saúde e serviços de apoio como, por exemplo, serventes, seguranças, entre outros. Já entre os profissionais de saúde, os Profissionais de Enfermagem, protagonistas deste estudo, configuram, aproximadamente, 2,2 milhões no Brasil, atuando em diferentes regiões e em proporções desiguais (COFEN, 2020 apud MIRANDA, et al., 2020). São profissionais que, independentemente do tipo de atendimento e do estado de saúde, atuam na linha de frente do cuidado prestado (MIRANDA, et al., 2020).

A essência da profissão dos enfermeiros é um processo de cuidar. Este processo não se limita ao desenvolvimento de atividades técnicas; também envolve conhecimento científico, sentimentos e emoções. O constante cenário de morte e estresse vivenciados no ambiente de trabalho e, na maioria das vezes, sobrecarregados de pacientes com forte capacidade de transmissão do vírus, necessita de um atendimento de enfermagem cuidadoso e rigoroso, incluindo procedimentos técnicos durante a paramentação e desparamentação, seguindo o que é recomendado cientificamente (MIRANDA et al., 2020).

Conforme o Boletim Epidemiológico Especial no i6 divulgado em maio no Brasil, no e-SUS Notifica foram cadastrados 199.768 profissionais de saúde, dos quais 31.790 (15,9\%) foram confirmados com a doença, 53.677 (26,9\%) foram descartados por não atenderem às normas laboratoriais clínicas e II4.30I (57,2\%) ainda estão sob investigação (PRADO et al., 2020). “A categoria profissional com o maior número de registros no sistema foi “Técnico ou Auxiliar em Enfermagem” (68.250 ou 34,2\%), seguida de "Enfermeiro" (33.733 ou 16,9\%), "Médico" (26.546 ou 13,3\%), "Recepcionista" (8.61o ou 4,3\%) e "Outro tipo de agente de saúde" (5.013 ou 2,5\%)" (MINISTÉRIO DA SAÚDE, 2020, p. Io). 
Devido ao rápido aumento do número de profissionais de saúde infectados com Covid-ı, bem como a toda exposição ao estresse e pressão, a saúde mental desses profissionais foi identificada como algo que requer uma atenção especial (PRADO et al., 2020). Além disso, o cansaço físico e mental, a dor de perder pacientes e colegas, a dificuldade na tomada de decisões, o medo da contaminação e a propagação da doença aos familiares, também são fatores que prejudicam a saúde mental dos profissionais de saúde atuantes na linha de frente no combate à doença (GUIMARÃES; BRASIL, 2018).

Nesse sentido, a presente pesquisa tem o intuito de compreender como o enfrentamento da pandemia de Covid-ı́ afeta a saúde mental dos profissionais de enfermagem.

\section{METODOLOGIA}

O presente estudo é uma revisão integrativa, que tem como principal característica uma ampla abordagem de metodologias referentes às revisões

bibliográficas em diferentes rotas de desenvolvimento, propiciando uma análise completa do objeto de pesquisa (SOUZA et al., 2010).

Para a estruturação deste trabalho, a coleta de informações foi realizada a partir da base de dados SciELO e das plataformas Google Scholar, Ministério da Saúde e Conselho Federal de Enfermagem, tendo como questão norteadora: "qual a percepção do profissional de enfermagem acerca de sua saúde mental no ambiente de trabalho no enfrentamento da pandemia da Covid-ı9?"

Como primeiro critério de inclusão foram selecionados artigos que estivessem relacionados à saúde mental dos profissionais de enfermagem no contexto da Covid19, publicados entre os anos 2020-2021, nos idiomas inglês e português, que contivessem em seus títulos: "Profissionais de enfermagem", "Enfermagem”, "Saúde mental", "Covid-I9", "Pandemia", "Mental health", "Nursing workers", "Nurse” e "Pandemic". 
A partir disso, foram escolhidos 27 estudos que se encaixavam no primeiro critério de inclusão. Como segundo critério, os resumos foram analisados, restando 13 estudos que permaneceram dentro da proposta de investigação, sendo estes lidos integralmente. A questão norteadora foi imprescindível para o desenvolvimento da análise, delimitando os textos produzidos sobre o assunto.

\section{RESULTADOS}

Seguindo os critérios de inclusão, foram listados abaixo (quadro I) os artigos que foram selecionados e analisados integralmente.

Quadro r: Artigos analisados

\begin{tabular}{|c|c|c|}
\hline TÍTULOS & AUTORES & ANO \\
\hline $\begin{array}{l}\text { Impactos da pandemia da } \\
\text { Covid- I9 na saúde } \\
\text { mental dos profissionais } \\
\text { de enfermagem }\end{array}$ & BARROS, A. B. et al. & 2020 \\
\hline $\begin{array}{l}\text { O impacto da pandemia } \\
\text { da Covid- I9 na saúde } \\
\text { mental dos profissionais } \\
\text { de saúde: revisão } \\
\text { integrativa }\end{array}$ & BEZERRA, G. et al. & 2020 \\
\hline $\begin{array}{l}\text { A saúde mental dos } \\
\text { profissionais de } \\
\text { enfermagem na linha de } \\
\text { frente à assistência de } \\
\text { paciente com Covid- I9 }\end{array}$ & $\begin{array}{c}\text { ALMEIDA, A. M. S. et } \\
\text { al. }\end{array}$ & $202 \mathrm{I}$ \\
\hline
\end{tabular}




\begin{tabular}{|c|c|c|}
\hline $\begin{array}{l}\text { A Covid- ig e o medo que } \\
\text { afeta a saúde mental dos } \\
\text { profissionais de } \\
\text { enfermagem: revisão de } \\
\text { literatura }\end{array}$ & MACEDO, J. M. da et al. & 2021 \\
\hline $\begin{array}{l}\text { O impacto da Covid- } 19 \\
\text { na saúde mental dos } \\
\text { profissionais de } \\
\text { enfermagem: revisão } \\
\text { integrativa }\end{array}$ & $\begin{array}{c}\text { CAMPIDELL, E. D. S. et } \\
\text { al. }\end{array}$ & 2021 \\
\hline $\begin{array}{l}\text { Decline in the mental } \\
\text { health of nurses of across } \\
\text { the globe during Covid- } \\
\text { I9: a systematic review } \\
\text { and meta-analysis }\end{array}$ & VARGHESE, A. et al. & 2021 \\
\hline $\begin{array}{l}\text { Aspectos inerentes à } \\
\text { saúde mental do } \\
\text { enfermeiro no combate a } \\
\text { pandemia da Covid- I9 }\end{array}$ & SILVA, R. G. et al. & 2021 \\
\hline Saúde mental e & & \\
\hline
\end{tabular}




\begin{tabular}{|c|c|c|}
\hline $\begin{array}{c}\text { intervenções psicológicas } \\
\text { diante da pandemia do } \\
\text { novo coronavírus } \\
\text { (COVID-rg) }\end{array}$ & SCHMIDT, B. et al. & 2020 \\
\hline $\begin{array}{l}\text { The Covid- } 19 \text { crisis: a } \\
\text { mental health perspective } \\
\text { and response using } \\
\text { telemedicine }\end{array}$ & SULLIVAN, A. B. et al. & 2020 \\
\hline $\begin{array}{c}\text { Resolução Cofen no } 634 \\
\text { Autoriza e normatiza a } \\
\text { teleconsulta de } \\
\text { enfermagem como forma } \\
\text { de combate à pandemia } \\
\text { provocada pelo novo } \\
\text { coronavírus }\end{array}$ & $\begin{array}{c}\text { RESOLUÇÃO COFEN } \\
\text { № } 634\end{array}$ & 2020 \\
\hline $\begin{array}{l}\text { Profissionais do SUS já } \\
\text { podem contar com } \\
\text { suporte psicológico }\end{array}$ & $\begin{array}{l}\text { MINISTÉRIO DA } \\
\text { SAÚDE }\end{array}$ & 2020 \\
\hline $\begin{array}{c}\text { Saúde mental dos } \\
\text { profissionais de } \\
\text { enfermagem durante a } \\
\text { pandemia de Covid- I9: }\end{array}$ & $\begin{array}{c}\text { RAMOS- TOESCHER, } \\
\text { A. M. et al. }\end{array}$ & 2020 \\
\hline
\end{tabular}




\begin{tabular}{|c|c|c|}
\hline recursos de apoio & & \\
\hline Saúde mental dos & & \\
profissionais de & & \\
enfermagem do Brasil no & HUMEREZ, D. C. et al. & \\
contexto da pandemia & HO2o \\
Covid- ig: ação do & & \\
Conselho Federal de & & \\
Enfermagem & & \\
\end{tabular}

\section{DISCUSSÃO}

O novo Coronavírus chegou de forma avassaladora, promovendo grandes momentos de tensão nos serviços de saúde. Surgindo, dessa maneira, vários fatores estressantes altamente capazes de afetar o bem-estar dos trabalhadores da área (BARROS et al., 2020). Com o advento da pandemia, a atenção encontrava-se inteiramente voltada para o tratamento da Covid- 19, passando despercebido os efeitos colaterais psicológicos causados por ela, principalmente entre os profissionais de enfermagem, cujo maior parte do tempo se encontra juntos dos pacientes, sendo, nesse contexto, um dos maiores protagonistas do atendimento aos atingidos pela doença (BARBOSA et al., 2020 apud BARROS et al., 2020).

Todavia, com o número de casos aumentando diariamente, tornou- se notória a necessidade de uma atenção especial com a saúde mental dos trabalhadores de enfermagem. Por se tratar de um desafio relativamente novo, esses profissionais enfrentam um ambiente instável, carente de infraestrutura adequada, falta de equipamentos de proteção individual (EPI's), inexperiência e insegurança, gerando altos níveis de desgaste físico e psicológico, reduzindo a qualidade de vida 
profissional e da assistência prestada (BEZERRA et al., 2020; ALMEIDA et al., 202ı; VARGHESE et al., 2021).

Sendo atuantes na linha de frente no combate à Covid- 19, as equipes de enfermagem se encontram mais suscetíveis a contaminação, devido à alta exposição a agentes biológicos, exaustivas jornadas de trabalho e grande responsabilidade diante do desconhecido (CAMPIDELL, OLIVEIRA \& FREITAS, 202I). De acordo com Silva et al. (2021) e Macedo, Souza \& Jesus (202I), nesse cenário os efeitos colaterais mais prevalentes são estresse, ansiedade, fadiga devido extensas jornadas de trabalho, frustrações, medo de serem contaminados e contaminar outras pessoas, principalmente familiares e colegas de trabalho, solidão e luto.

Em vista disso, as ações direcionadas à saúde psicológica desses profissionais tem exercido um papel essencial. Destacam- se, dessa maneira, uma série de atendimentos especializados através canais de informação e comunicação, que consistem em métodos pertinentes para o acolhimento associados à saúde mental (SCHIMIDT et al., 2020), com o intuito de amenizar o estresse e intenso sofrimento, prevenindo maiores danos no futuro.

Os impactos da pandemia na saúde mental, evidenciaram a importância da implementação de sistemas de atenção biopsicossocial, sendo indicadas desde o primeiro momento. $O$ atendimento de forma remota é uma opção, que além de reduzir a disseminação do vírus, fornece apoio emocional respeitando as recomendações de distanciamento feitas pela a OMS (DUAN et al., 2020 apud RAMOS-TOESCHER et al., 2020).

A telessaúde, mais especificamente a telepsicologia, são meios de atendimento remoto já existentes desde antes de o surto da Covid-ı, que ganharam força e se tornaram fundamentais para atender as necessidades decorrentes da pandemia (SULLIVAN et al., 2020). O Conselho Federal de Enfermagem (COFEN), em 2020, colocou no ar um canal de atendimento 24 horas coordenado por enfermeiros especializados, designado a todos os trabalhadores de enfermagem que precisarem de assistência psicológica durante esse período (RESOLUÇÃO COFEN no 634, março 
de 2020; HUMEREZ, OHL \& SILVA, 2020). O Ministério da Saúde também investiu no atendimento remoto, como o canal TeleSUS, formado por psiquiatras e psicólogos, que dependendo da complexidade das intervenções, devem encaminhar para atendimento presencial (MINISTÉRIO DA SAÚDE, 2020).

Diante disso, é inegável o quanto essas plataformas se tornaram indispensáveis na manutenção da saúde psicológica e emocional desses profissionais que lidam diariamente com a pressão e as incertezas causadas pela pandemia da Covid-19. Portanto, através dos meios digitais é possível promover a saúde e prevenir agravos psicoemocionais, oferecendo apoio e orientação de como lidar com essa situação tão desafiadora (SCHIMIDT et al., 2020).

\section{CONSIDERAÇÕES FINAIS}

Com a chegada da Covid- 19 e o pouco conhecimento sobre ela, colocou- se em evidência um problema recorrente entre os profissionais de enfermagem, a saúde mental. A proximidade com os pacientes nesse cenário pandêmico desencadeia uma série de eventos estressantes, gerando ansiedade, depressão, solidão devido ao distanciamento social, medo de se contaminar e contaminar familiares, entre outros.

Nesse contexto, esses profissionais, mais que nunca, necessitam de atenção direcionada à suas necessidades, como identificação de possíveis problemas, melhorias no ambiente de trabalho, acesso a apoio psicoemocional, capacitação adequada, fornecimento de EPI's, flexibilização nas extensas jornadas de trabalho e ambientes apropriados para descanso.

Contudo, é extremamente necessário enxergar o valor dos trabalhadores de enfermagem, principalmente em vista da situação vivenciada nos últimos dois anos. Por essa razão, além de meios adequados para o desenvolvimento do trabalho, a preservação da saúde mental e emocional desses profissionais, é indispensável para se promover qualidade de vida e qualidade na assistência prestada. 


\section{REFERÊNCIAS}

AlMEIDA, A. M. S.; LIMA, K. P.; SILVA, M. S. D.; CARVAlHO, S. T. A.; SILVA, B. D. DA; LEONARDO, B. L. P.; MARQUES, M. A. D. S. S.; SOUSA , J. DA C. A. DE; MELO, J. B. A.; CARVAlHO, A. L.; GOMES, M. R. DA S. A saúde mental dos profissionais de enfermagem na linha de frente à assistência de pacientes com covid-19. Revista de Casos e Consultoria, v. I2, n. I, p. e25073, i7 jun. 202I.

BARROS, A. B.; SILVA, V. R. da; GOMES, K. E. A.; MONTE, E. C.; MOURA, M. E. R. B. de; ALVES, S. M.; LIRA, P. F.; LUZ, D. C. R. P. IMPACTOS DA PANDEMIA DA COVID-ı NA SAÚDE MENTAL DOS PROFISSIONAIS DE ENFERMAGEM / IMPACTS OF THE COVID-I9 PANDEMIC ON THE MENTAL HEALTH OF NURSING PROFESSIONALS. Brazilian Journal of Development, [S.L.], v. 6, n. 1o, p. 81175-81184, 23 out. 2020. Brazilian Journal of Development. http://dx.doi.org/ro.34117/bjdv6nio-514. Disponível em: https://www.brazilianjournals.com/index.php/BRJD/article/view/r870o/15062.

Acesso em: 2I set. 2021. BEECHING N, FLETCHER T, FOWLER R, BMJ Best Practice. Apr 202I, https://bestpractice.bmj.com/topics/pt-br/300020I. Last accessed 29th April 2021.

BEZERRA, G.; SENA, A. S.; BRAGA, S.; DOS SANTOS, M. E.; CORREIA, L. F.; CLEMENTINO, K. M.; CARNEIRO, Y. V.; PINHEIRO, W. O impacto da pandemia por COVID-ı9 na saúde mental dos profissionais de saúde: revisão integrativa. Revista Enfermagem Atual In Derme, v. 93, p. e-020or2, 4 set. 2020.

BIBLIOTECA VIRTUAL EM SÁUDE. Brasil: Novo Coronavirus (Covid-I9): Informações Básicas, 2020. Disponível em: https://bvsms.saude.gov.br/novocoronavirus-covid-I9-informacoes-basicas/. Acesso em: 04 out. 202I.

CAMPIDELL, E. D. S.; OLIVEIRA, F. C. P.; FREITAS, P. A. de. O impacto da pandemia COVID-19 na saúde mental da enfermagem: Revisão Integrativa. 2021. I5 f. TCC (Graduação) - Curso de Enfermagem, Centro Universitário Una, Barreiro, 2021. Disponível em: https://repositorio.animaeducacao.com.br/handle/ANIMA/I47or. Acesso em: 2I set. 2021.

GUIMARÃES, A. V.; BRASIL, A. M. O ADOECIMENTO PSÍQUICO E A ATIVIDADE LABORAL DO PROFISSIONAL DE SAÚDE. 2018. 26 f. TCC (Doutorado) - Curso de Psicologia, Centro Universitário de Anápolis Unievangélica, 
Anápolis, 2018. Disponível em: http://repositorio.aee.edu.br/jspui/handle/aee/IIzo. Acesso em: 30 mar. 202I.

HUMEREZ, D. C. de; OHL, R. I. B.; SILVA, M. C. N. da. SAÚDE MENTAL DOS PROFISSIONAIS DE ENFERMAGEM DO BRASIL NO CONTEXTO DA PANDEMIA COVID-rg: AÇÃO DO CONSELHO FEDERAL DE ENFERMAGEM. Cogitare Enfermagem, [S.1.], v. 25, maio 2020. ISSN 2176-9133. Disponível em: 〈https://revistas.ufpr.br/cogitare/article/view/74115/40808〉. Acesso em: 21 set. 2021. doi:http://dx.doi.org/10.5380/ce.v25io.74115.

MACEDO, . J. M. da S. .; SOUZA, R. C. da S. .; JESUS, . A. L. S. de. A COVID-i9 E O MEDO QUE AFETA A SAÚDE MENTAL DOS PROFISSIONAIS DE ENFERMAGEM: REVISÃO LITERÁRIA. Revista JRG de Estudos Acadêmicos , [S. l.], v. 4, n. 9, p. 58-65, 2021. DOI: 10.528I/zenodo.5093816. Disponível em: https://revistajrg.com/index.php/jrg/article/view/278. Acesso em: 2I set. 202I.

MINISTÉRIO DA SAÚDE (BR). Profissionais do SUS já podem contar com suporte psicológico [Internet]. Brasília; 2020 [citado 2021 Setembro 26]. Disponível em: https://www.saude.gov.br/noticias/agencia-saude/469o6-profissionais-do-susja-podem-contar-com-suporte-psicologico

MINISTÉRIO DA SAÚDE. Secretaria de Vigilância Sanitária em Saúde. Boletim Epidemiológico Especial Ni6: Centro de Operações de Emergência em Saúde Pública pela Doença do Coronavírus 2019 (COE-COVIDi9). 2020. Disponível em: https://portalarquivos.saude.gov.br/images/pdf/2020/May/2I/2020-05-19---BEEı6--Boletim-do-COE-rzh.pdf

MIRANDA, F. M. D.; SANTANA, L de L.; PIZZOLATO, A. C.; SARQUIS, L. M. M. CONDIÇÕES DE TRABALHO E O IMPACTO NA SAÚDE DOS PROFISSIONAIS DE ENFERMAGEM FRENTE A COVID-19. Cogitare Enfermagem, [S.1.], v. 25, may 2020. ISSN 2176-9133. Disponível em: 〈https://revistas.ufpr.br/cogitare/article/view/72702〉. Acesso em: I8 mar. 2021. doi:http://dx.doi.org/I0.538o/ce.v25io.72702.

OLIVEIRA, W. K. de; DUARTE, E.; FRANÇA, G. V. A.; GARCIA, L. P. Como o Brasil pode deter a COVID-ı. Epidemiologia e Serviços de Saúde [online]. v. 29, n. 2 [Acessado 17 Março 2021], e2020044. Disponível em: <https://doi.org/I0.5123/Si679- 
49742020000200023>. mental dos profissionais de saúde frente à pandemia do COVID-I9: uma revisão integrativa. Revista Eletrônica Acervo Saúde, n. 46, p. e4128, 26 jun. 2020.

RAMOS- TOESCHER, A. M.; TOMASCHEWISK- BARLEM, J. G.; BARLEM, E. L. D.; CASTANHEIRA, J. S.; TOESCHER, R. L. Saúde mental de profissionais de enfermagem durante a pandemia de COVID-ig: recursos de apoio. Escola Anna Nery [online]. 2020, v. 24, n. spe [Acessado I Setembro 2021] , e20200276. Disponível em: 〈https://doi.org/10.1590/2177-9465-EAN-2020-0276〉. Epub i9 Out 2020. ISSN 2177-9465. https://doi.org/10.1590/2177-9465-EAN-2020-0276.

RESOLUÇÃO COFEN no 634, de 26 de março de 2020 (BR). Autoriza e normatiza a teleconsulta de enfermagem como forma de combate à pandemia provocada pelo novo coronavírus (Sars-Cov-2). Diário Oficial da União, Brasília (DF), 27 mar 2020 [citado 2021 Setembro 26]. Disponível em: http://www.cofen.gov.br/resolucaocofen-no-o634-2020_78344.html

SCHMIDT, B.; CREPALDI, M. A.; BOLZE, S. D. A.; NEIVA- SILVA, L.; DEMENECH, L. M. Saúde mental e intervenções psicológicas diante da pandemia do novo coronavírus (COVID-19). Estudos de Psicologia (Campinas) [online]. 2020, v. 37 [Acessado 26 Setembro 202I], ezooo63. Disponível em: 〈https://doi.org/10.1590/1982-0275202037e200063〉. Epub I8 Maio 2020. ISSN 19820275. https://doi.org/10.1590/1982-0275202037e200063.

SILVA, R. G.; PINTO, W. M.; RODRIGUES, K. N.; BOTELHO, M. S. L.; BATISTA, M. H de J. Aspectos inerentes à saúde mental do enfermeiro no combate a Pandemia da COVID-ı. Brazilian Journal of Health Review, [S.L.], v. 4, n. 4, p. I5471-15485, 20 jul. 2021. South Florida Publishing LLC. http://dx.doi.org/ro.34119/bjhrv4n4-o86. Disponível em: https://www.brazilianjournals.com/index.php/BJHR/article/view/33183. Acesso em: 2I set. 202I.

SUllivan, A. B.; KANE, A.; ROTH, A. J.; DAVIS, B. E.; DRERUP, M. L.; HEINBERG, L. J.; The COVID-rg Crisis: a mental health perspective and response using telemedicine. Journal Of Patient Experience, [S.L.], v. 7, n. 3, p. 295-30I, II 
maio 2020. SAGE Publications. http://dx.doi.org/Io.II77/2374373520922747. Disponível em: https://journals.sagepub.com/doi/ro.1177/2374373520922747. Acesso em: 26 set. 2021.

VARGHESE, A.; GEORGE, G.; KONDAGULI, S.; NASER, A. Y.; KHAKHA, D. C.; CHTTERJI, R. Decline in the mental health of nurses across the globe during COVID-I9: a systematic review and meta-analysis. Journal Of Global Health, [S.L.], v. II, p. I-I5, Io abr. 202I. International Global Health Society. http://dx.doi.org/ro.7189/jogh.II.05009. Disponível em: https://pubmed.ncbi.nlm.nih.gov/33884193/. Acesso em: 21 set. 2021.

WERNECK, G. L. e CARVALHO, M. S. A pandemia de COVID-í no Brasil: crônica de uma crise sanitária anunciada. Cadernos de Saúde Pública [online]. v. 36, n. 5 [Acessado I7 Março 202I], eooo68820. Disponível em: 〈https://doi.org/ı.1590/oro2-311Xooo68820〉. ISSN https://doi.org/10.1590/oro2-311Xooo68820 